Portland State University

PDXScholar

\title{
A Computer Model of Intracranial Pressure Dynamics During Traumatic Brain Injury that Explicitly Models Fluid Flows and Volumes
}

\author{
Wayne W. Wakeland \\ Portland State University, wakeland@pdx.edu \\ Brahm Goldstein \\ Oregon Health \& Science University \\ Louis Macovsky \\ Dynamic BioSystems \\ James McNames \\ Portland State University, mcnames@pdx.edu
}

Follow this and additional works at: https://pdxscholar.library.pdx.edu/sysc_fac

Part of the Analytical, Diagnostic and Therapeutic Techniques and Equipment Commons, Congenital, Hereditary, and Neonatal Diseases and Abnormalities Commons, and the Disease Modeling Commons Let us know how access to this document benefits you.

\section{Citation Details}

Wakeland, Wayne W.; Goldstein, Brahm; Macovsky, Louis; and McNames, James, "A Computer Model of Intracranial Pressure Dynamics During Traumatic Brain Injury that Explicitly Models Fluid Flows and Volumes" (2003). Systems Science Faculty Publications and Presentations. 19.

https://pdxscholar.library.pdx.edu/sysc_fac/19

This Technical Report is brought to you for free and open access. It has been accepted for inclusion in Systems Science Faculty Publications and Presentations by an authorized administrator of PDXScholar. Please contact us if we can make this document more accessible: pdxscholar@pdx.edu. 


\title{
A COMPUTER MODEL OF INTRACRANIAL PRESSURE DYNAMICS DURING TRAUMATIC BRAIN INJURY THAT EXPLICITLY MODELS FLUID FLOWS AND VOLUMES
}

\author{
Wayne Wakeland \\ Biomedical Signal Processing Laboratory, Department of Electrical Engineering, Portland State \\ University \\ Systems Science Ph.D. Program, Portland State University \\ wakeland@pdx.edu \\ Technical Report 03-01
}

April 6, 2003

\begin{abstract}
This report documents a computer model of intracranial pressure (ICP) dynamics that is used to evaluate clinical treatment options for elevated ICP during traumatic brain injury (TBI). The model uses fluid volumes as primary state variables and explicitly models fluid flows as well as the resistance, compliance, and pressure associated with each of the compartments (arteries and arterioles, capillary bed, veins, venous sinus, ventricles, and brain parenchyma). The model has been tested to assure that it reproduces a correct physiologic response to intra- and extra-parenchymal hemorrhage and edema, and to therapies directed at reducing ICP such as cerebral spinal fluid drainage, mannitol administration, head elevation, and mild hyperventilation.
\end{abstract}

The model is able to replicate observed clinical behavior in many cases, including elevated ICP associated with severe cerebral edema, subdural hematoma, and cerebrospinal fluid blockage. The model also successfully reproduces the cerebrovascular regulatory mechanisms that are activated during TBI in response to various abnormalities such as high or low systemic blood pressure.

We conclude that incorporating fluid volumes and flows into a model of ICP dynamics significantly improved its clinical utility. Additional improvements are anticipated (or will accrue or will result) as the specific mechanisms that modify cerebral compliance and autoregulation during TBI and elevated ICP are further delineated.

\section{INTRODUCTION}

Our ICP dynamic model is similar in many ways to other dynamic ICP models found in the literature (Czosnyka 1997; Ursino 1995; Ursino 1997) in that it considers the cranial vault to contain the brain parynchema plus several fluid compartments that together are constrained not to exceed the total cranial volume (i.e, the Monro-Kellie hypothesis). The compartments include the arterial blood volume, capillary blood volume, venous blood volume, CSF volume, the brain volume, and "other" volume (such as an epidural hematoma). For many of scenarios, brain volume remains constant, as it is in other models. However, in some scenarios, especially those associated with certain types of TBI, the assumption of fixed brain volume is not accurate. Our model allows, for example, for cerebral edema to be modeled.

As with previously published models, cerebral autoregulation is modeled as a feedback loop that causes the vasculature to dilate or constrict, taking into account control limits that are non-linear and asymmetric. Our control logic acts only on the flow of blood from the arterial compartment to the capillary bed. This approximation will be evaluated during this project. The control logic is proportional and has enough "gain" that it can easily maintain the required flow under normal conditions. However, if the venous or arterial volumes are severely reduced, as is often the case with TBI, the associated non-linear increases in resistance can "overwhelm" the control logic (loss of cerebral autoregulation). 
The model differs from the other ICP models because, rather than directly modeling the pressure in each of the compartments as a state variable, we have modeled the actual fluid volumes as state variables, and have also explicitly modeled the fluid flows into and out of each compartment. This approach is more intuitive, and it is easier to represent the relevant pathophysiology. Blood pressures are computed from the blood volumes and the arterial and venous compliances. Cerebral spinal fluid pressure is computed using the total intracranial volume and the pressure volume index (PVI) (Marmarou 1978). Our ICP dynamic model is not strictly consistent with the Monro-Kellie hypothesis that total intracranial volume ([brain volume ] + [blood volume] + [cerebral spinal fluid volume] + ["other" volume]) cannot change. In our model, small increases in the total intracranial volume do occur, which causes ICP to increase as indicated by the PVI (Marmarou 1978).

The model was developed using the STELLA (STELLA 2003) simulation language because: 1) it is well suited to the formulation phase of dynamic modeling, 2) it is easy to represent the flows and storage of fluids, and 3) model structure can be relatively easily reviewed and understood by non-mathematicians.

The model has been designed to have the capability to reproduce the dynamic behavior associated with the multiple types of TBI pathophysiology, including epidural hematoma, subdural hematoma, intraparynchemal hemorrhage, focal or generalized cerebral edema, and depressed skull fracture. Many previously published ICP models allowed for only a limited number of pathophysiologic scenarios. Our model allows for any combination of these pathophysiologies that are may be encountered in clinical practice. For example, the response of our model to a simulated epidural hematoma, includes focal cerebral edema and elevated ICP that is frequently observed in clinical situations.

The model also takes into account different time constants for development of hemorrhage depending on the source of the bleeding (venous vs. arterial vs. capillary). In addition, our model is also able to incorporate common treatment modalities such as intravenous mannitol, elevating the head of the bed to $30^{\circ}$, and mild hyperventilation (with decreased $\mathrm{PaCO}_{2}$ ), and cerebral spinal fluid drainage.

A simplified diagram of our initial ICP dynamic model is shown in Figure 1.

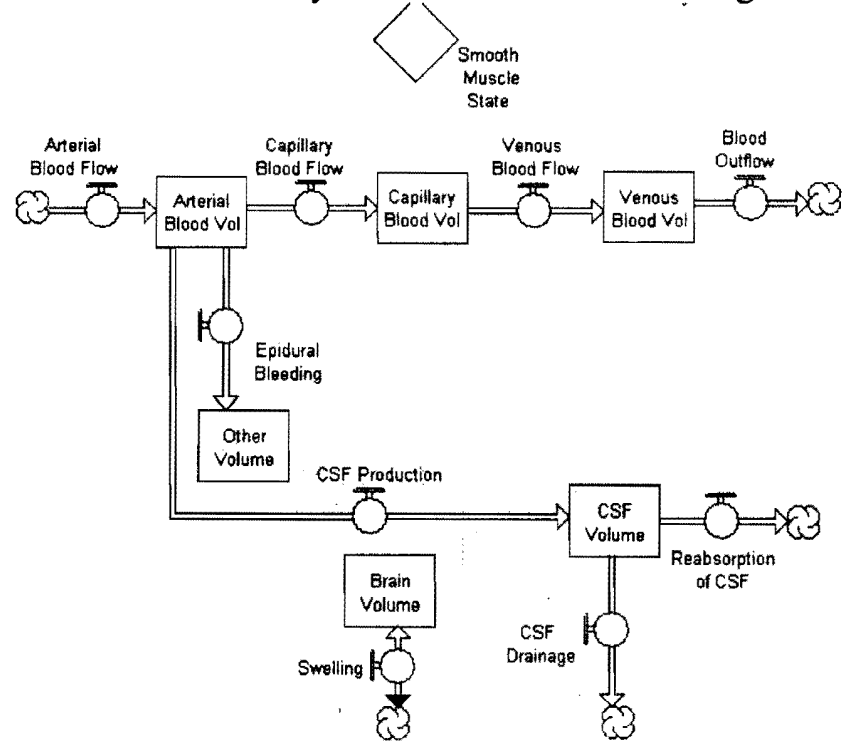

Figure 1. Overview of ICP Dynamic Mode.I 


\section{MODEL BEHAVIOR}

The model is able to replicate many aspects of the behavior associated with TBI. Figure 2 shows an example simulation run four minutes in duration. During the first minute, the model indicates dynamic equilibrium. From 1 minute to 1 minute 30 seconds, a $25 \mathrm{~mL}$ epidural hematoma is simulated. This caused ICP to increase, and venous blood was forced out of the cranial vault. At 2 minutes, CSF fluid drainage is simulated, to reduce ICP. This model qualitatively reproduces the pathophysiology associated with increased ICP associated with a rapid epidural hemorrhage.

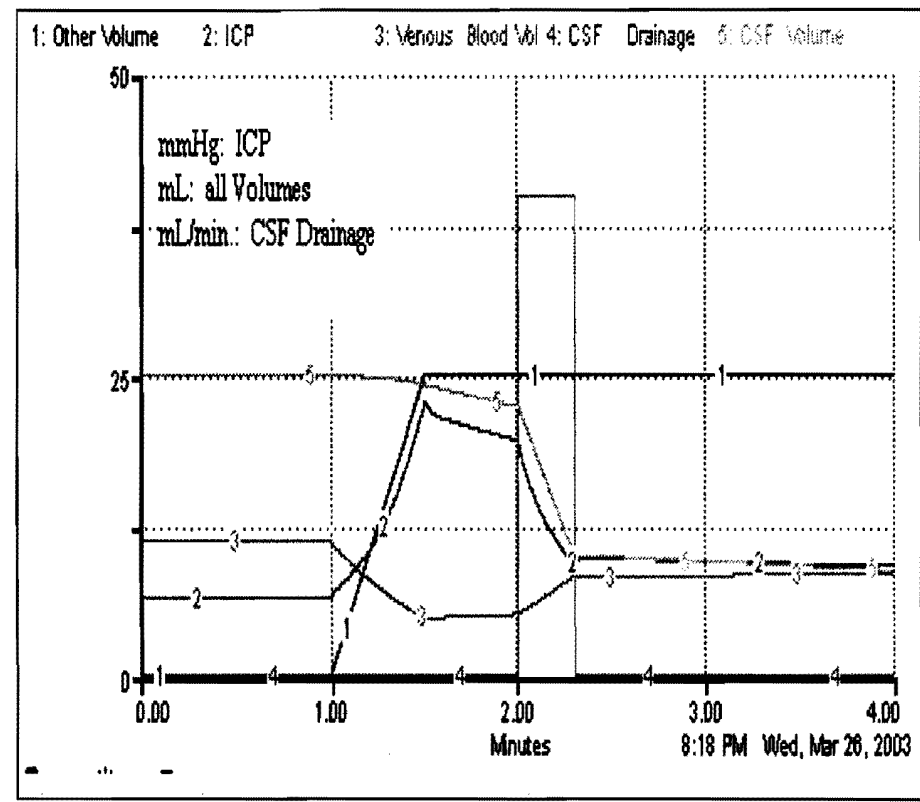

Figure 2: Sample Simulation Run of the Preliminary ICP Dynamic Model ( $y$ axis units $=\mathrm{mmHg}$ )

\section{CLINICAL REFERENCE DATA}

An example of the clinical behavior that must be reproduced by the model is patient response to CSF drainage, one of the common therapies for reducing elevated ICP.

Data covering three episodes of CSF drainage were obtained from the Complex Systems Laboratory (CSL 2003), including 5 minutes prior to the drainage and 15 minutes afterwards. Since the signal integrity is compromised when the drain is first opened, a small segment of data is intentionally omitted at the point when the drainage was initiated. The data was sampled at $125 \mathrm{~Hz}$. MATLAB was used to low pass filter the data and then upsample it (via decimation) in order to provide 75 sample points per minute. The data was exported to an ASCII file to facilitate comparison with model results. Figure 3 shows this data in the time domain for three specific episodes. This data will be compared later in this proposal to preliminary model results. 


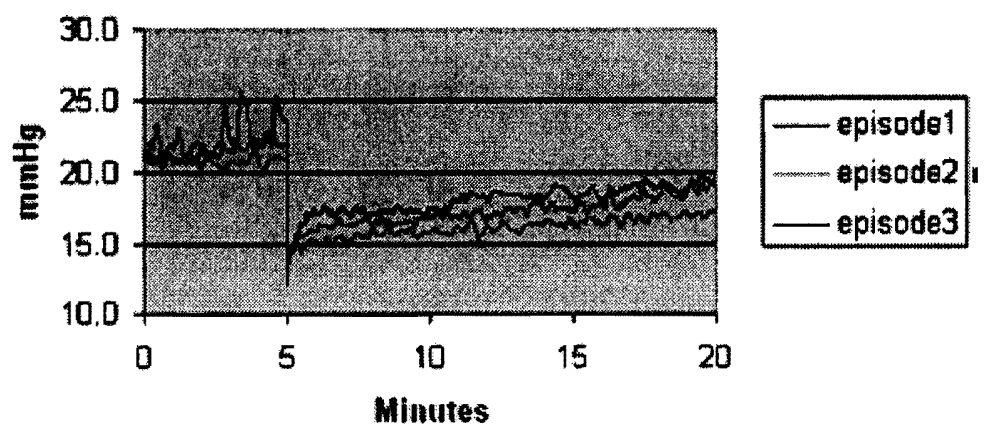

Figure 3: Sample ICP data before and after CSF drainage

\section{CALIBRATION OF MODEL TO MATCH REFERENCE DATA}

The model was recalibrated to approximate the actual clinical results associated with the data in Figure 3. In order to replicate this behavior, the model estimated a $24 \mathrm{~mL}$ epidural hematoma, drainage of $6.5 \mathrm{~mL}$ of cerebral spinal fluid, plus an increase in the resistance to CSF uptake, such as that seen with blockage of CSF circulation or re-absorption. Figure 4 shows the ICP predicted by model versus a plot of the average of the three clinical episodes.

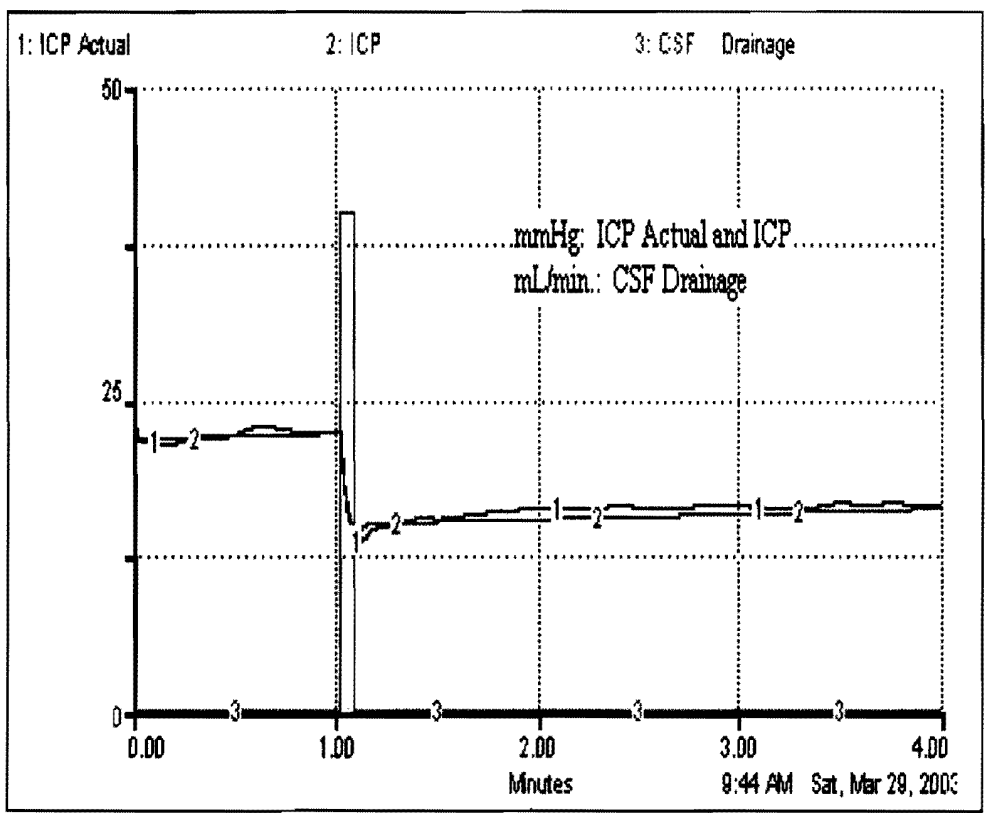

Figure 4: ICP before and after CSF drainage at 1 minute, Model vs. Actual Data ( $y$ axis units $=\mathrm{mmHg}$ )

There is no way to be certain of the exact pathophysiologic changes that caused this patient's elevated ICP, but our model enables exploration of potential etiologies. Note how closely the model (blue) correlates with the actual (red) ICP data.

\section{MODEL DETAILS}

The full "flow diagram" for the model is shown in Figure 5. We recognize that many readers may not be familiar with this type of diagram. The rectangles represent the accumulations or 
compartments (of blood and CSF in this case). The double arrows represent fluid flows. In the middle of each double arrow is a symbol representing a valve. The thin arrows connecting into these valves indicate the information needed to determine the flow rate. In general, the thin arrows represent "information" linkages throughout the model. Circles represent additional equations or logic. For example, "Pa ic" is represented as a circle, indicating that it is an algebraic formula. Three arrows point into $\mathrm{Pa}$ ic, indicating that it is computed [instantaneously] from three other model components: Arterial Compliance, Arterial Blood Volume, and ICP. The diagram is detailed and complex, but the advantage of this complexity is that the logic is made very explicit.

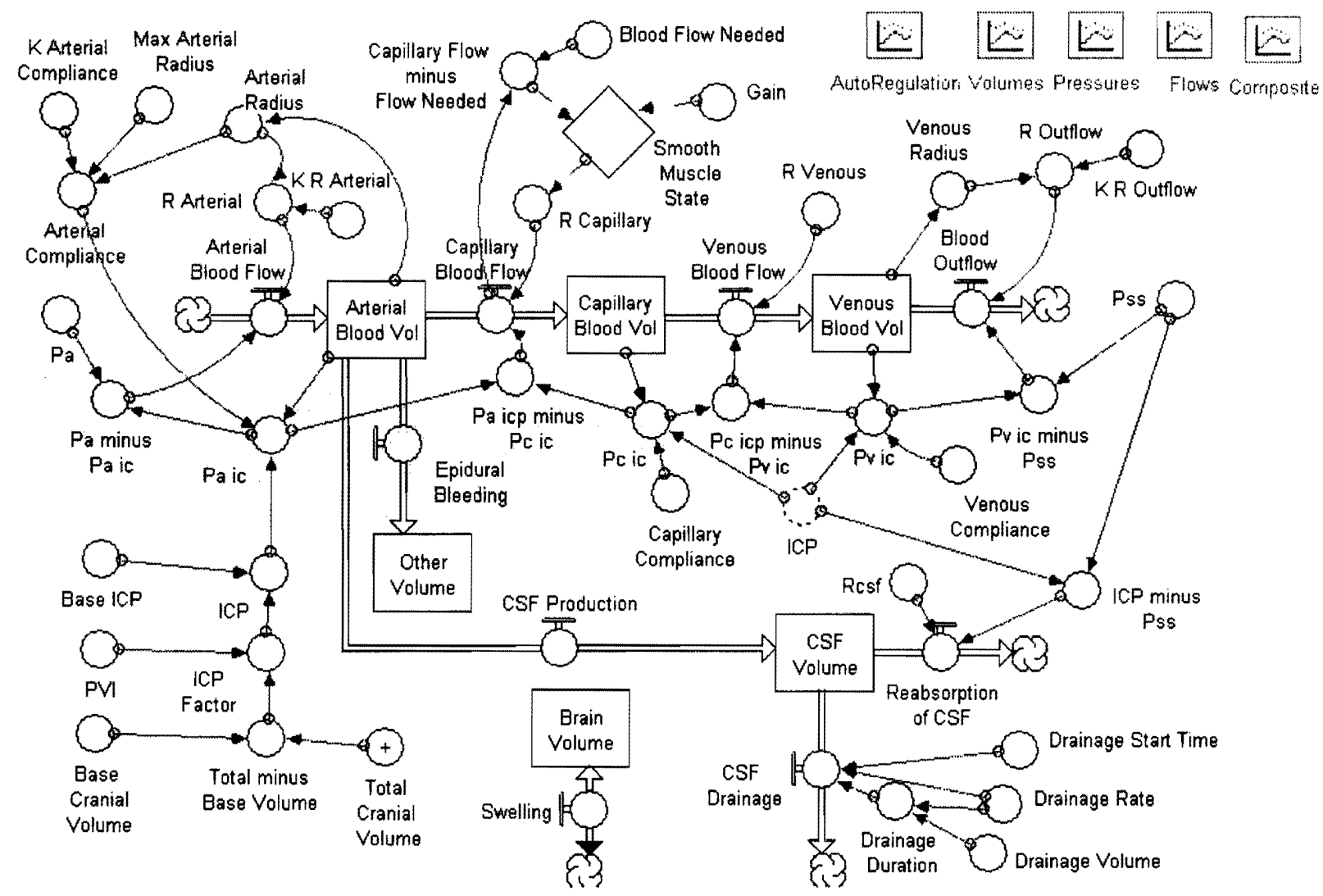

Figure 6. Structure of the ICP Dynamic Model. Abbreviations are as follows: CSF, cerebral spinal fluid; ICP, intracranial pressure; $\mathrm{K}$, constant; Max, maximum; $\mathrm{Pa}$, arterial blood pressure; $\mathrm{Pa}$ ic, intracranial arterial pressure; $\mathrm{Pc}$ ic, intracranial capillary pressure; $\mathrm{Pv}$ ic, intracranial ventricular pressure; Pss, saggital sinus pressure; PVI, pressurevolume index; $R$, resistance; $R$ arterial, arterial resistance; $R$ csf, resistance to CSF re-absorption. Other variable names are spelled out in order to more clearly indicate what they represent. Note that cerebral edema can be simulated by increasing "Brain Volume" with resultant "Swelling", an epidural hematoma can be simulated by increasing "Arterial Blood Volume" to "Other Volume", and any other single or combination of pathologies are able to be simulated using real-time data.

\section{FUTURE PLANS}

A. Implement the model logic in MATLAB/Simulink in order to take advantage of its greater flexibility and ability to process archived signal data.

B. Calibrate the model to correspond with data from specific patients or groups of patients. 
The pathophysiologic information for various patient groups will be represented in the model. Parameters specific to that particular pathophysiology will be optimized to fit the clinically annotated data showing how the patients responded to specific therapies, such as mannitol injection, elevating the head of the bead, and mild hyperventilation. For each patient/group, base parameter values will be determined for the compliance and resistance for each compartment, cerebral autoregulation characteristics, and the overall cranial pressure-volume index.

C. Incorporate improved measures of cerebral autoregulation and compliance into the model.

We model cerebral autoregulation as a feedback loop that causes the vasculature to dilate or constrict, taking into account control limits that are non-linear and asymmetric. This control logic acts only on the flow of blood from the arterial compartment to the capillary bed. This approximation will be evaluated during this project using physiologic signal data. As we develop improved measures of autoregulation and cerebral compliance, they will be incorporated into the model.

D. Thoroughly test the fully-calibrated model.

The fully-calibrated model will be tested thoroughly as prescribed in the System Dynamcis literature (c.f. Sterman 2000). These tests include:

1) Testing the sensitivity of the model to the full range of values for parameters, initial conditions, and external inputs observed in clinical situations, including pressures, flow rates, volumes, resistances, compliances, and cerebral autoregulatory gain and limits.

2) Taking parameter values to extremes in order to establish the points at which the model becomes obviously invalid.

3) Hypothesis testing, where changes to the model are contemplated, and hypotheses are stated regarding how the model behavior should change. The model is then run and the resulting behavior is compared to and reconciled with the hypothesis. The reconciliation could result in changes to the model or recognition that the intuitive hypothesis was not correct. This type of hypothesis testing is very different than traditional statistical hypothesis testing, but is very useful nonetheless. Both parametric and structural changes are considered and tested in this fashion.

4) Identifying and analyzing the feedback loops in the model. In the medical literature, feedback loops are often referred to as cascades. Feedback is present whenever changes at one point in the model cause a chain of events that lead back to starting point. A loop is classified as positive or reinforcing, when the impact of the chain of events is in the same direction as the initial change. A loop is considered negative or balancing when the impact of the change of events is opposite to the original change. Loops are also characterized in terms of their gain and their effective time constant. Feedback loops are explicitly analyzed in order to assure that their characteristics correspond to the known physiology.

5) Studying the stability characteristics of the model by conducting experiments to determine the combinations of parameters that result in stable versus unstable behavior. This is one more way to build confidence that the model corresponds to the known physiology.

\section{CONCLUSION}

The behavior of the model appears to be qualitatively correct, and we are beginning to calibrate the model quantitatively against carefully collected and annotated clinical data. Our preliminary 
results are encouraging and have been submitted for presentation at the $12^{\text {th }}$ International Symposium on Intracranial Pressure and Brain Monitoring (Wakeland 2003).

We are confident that we will be able to refine the model to accurately reflect the different pathophysiologies encountered in the clinical environment.

\section{REFERENCES}

CSL [Complex Systems Laboratory]: http://www.ohsuhealth.com/dch/complex. 3/23/03.

Czonyka M, Piechnik S, et al. Contribution of mathematical modeling to the interpretation of bedside tests of cerebrovascular autoregulation. J Neurosurg 1997;63:721-731.

Marmarou A, Shulman K, et al. A nonlinear analysis of compliance and outflow resistance of the cerebrospinal fluid system. $J$ Neurosurg 1978;48:332-344.

Rosner MJ. Pathophysiology and management of increased intracranial pressure. In: Neurosurgical Intensive Care, Andrew BT, McGraw-Hill, Inc, 1993: 57-112.

STELLA. http://www.hps-inc.com/STELLAVPSR.htm. 3/23/2003.

Sterman J. Business Dynamics: Systems Thinking and Modeling for a Complex World. Irwin/McGraw Hill, Boston, MA 2000.

Ursino M, Lezzi M, et al. Intracanial Pressure Dynamics in Patients with Acute Brain Damage: A Critical Analysis with the Aid of a Mathematical Model. IEEE Transactions on Biomedical Engineering 1995;42:529-540.

Ursino M, Lodi CA, et al. Intracranial pressure dynamics in patients with acute brain damage. $J$ Appl Physiol 1997;82:1270-1282.

Wakeland W, B. Goldstein, L. Macovsky, J. McNames. A Computer Model of Intracranial Pressure Dynamics during Traumatic Brain Injury that Explicitly Models Fluid Flows and Volumes. $12^{\text {th }}$ International Symposium on Intracranial Pressure and Brain Monitoring 2003 (submitted). 